\title{
Dual red imaging: a novel endoscopic imaging technology visualizing thick blood vessels in the gastrointestinal wall
}

\section{(ㄷ)(1) $\ominus$}

Authors

Naohisa Yahagi ${ }^{1}$, Ai Fujimoto ${ }^{1,2}$, Joichiro Horii ${ }^{3}$, Toshio Uraoka ${ }^{4}$, Masayuki Shimoda ${ }^{5}$, Kaoru Takabayashi ${ }^{6}$, Toshihiro Nisizawa ${ }^{2}$, Osamu Goto ${ }^{7}$, Yasutoshi Ochiai ${ }^{1}$, Tadateru Maehata ${ }^{1}$, Atushi Nakayama ${ }^{1}$, Motohiko Kato ${ }^{1,8}$, Naoki Hosoe ${ }^{6}$, Makoto Naganuma ${ }^{8}$

Institutions

1 Division of Research and Development for Minimally Invasive Treatment, Cancer Center, Keio University School of Medicine, Tokyo, Japan

2 Department of Gastroenterology, National Hospital Organization, Tokyo Medical Center, Tokyo, Japan

3 Department of Gastroenterology, National Hospital Organization, Fukuyama Medical Center, Fukuyama, Hiroshima, Japan

4 Department of Gastroenterology and Hepatology, Gunma University Graduate School of Medicine, Maebashi, Japan

5 Department of Pathology, Keio University School of Medicine, Tokyo, Japan

6 Center for Diagnostic and Therapeutic Endoscopy, Keio University School of Medicine, Tokyo, Japan

7 Department of Gastroenterology, Nippon Meidal School, Graduate School of Medicine Tokyo, Japan

8 Division of Gastroenterology and Hepatology, Department of Internal Medicine, Keio University School of Medicine, Tokyo, Japan

submitted 12.5.2018

accepted after revision 24.9.2018

\section{Bibliography}

DOI https://doi.org/10.1055/a-0749-0075 |

Endoscopy International Open 2019; 07: E1632-E1635

(c) Georg Thieme Verlag KG Stuttgart · New York eISSN 2196-9736

Corresponding author

Naohisa Yahagi, MD PhD, 35 Shinanomachi, Shinjuku-ku, Tokyo 160-8582, Japan
Fax: +81-3-53633895

yahagi-tky@umin.ac.jp

\section{ABSTRACT}

Background Dual red imaging (DRI), a novel image-enhanced endoscopy (IEE) technology, has the potential to improve the visibility of blood vessels in deeper tissue using $600 \mathrm{~nm}$ and $630 \mathrm{~nm}$ wavelength lights in the red band.

Aim To confirm the feasibility of DRI in visualization of vessels in deeper tissue and identify pathologically the features of blood vessels visualized by DRI.

Methods Study 1: visibility of blood vessels was assessed by five observers in 137 pairs of DRI and white light imaging (WLI) images. The scores for the visibility of thick blood vessels were measured for randomized images and compared with the scoring template as a reference. The difference in visibility score between DRI and WLI was compared in each pair of images. Study 2: blood vessels detected only by DRI were examined pathologically using two pig stomachs.

Results Study 1: The mean visibility scores of DRI and WLI for each observer were $1.69-2.26$ and $1.31-1.67$, respectively. The mean difference in visibility score and $95 \%$ confidence interval for the five observers was 0.59 [0.46$0.72], 0.54$ [0.40-0.68], 0.34 [0.18-0.49], 0.51 [0.36$0.66]$, and $0.71[0.54-0.88]$. The visibility was statistically significantly better in DRI than in WLI for all observers $(P<$ $0.0001)$. Study 2 : three blood vessels were observed only by DRI. All of these blood vessels were located at a depth of $1000-1500 \mu \mathrm{m}$ from the mucosal surface. The diameter of these blood vessels exceeded $80-200 \mu \mathrm{m}$.

Conclusions DRI can feasibly detect thick blood vessels in the deep mucosa or submucosa of the gastrointestinal tract.

\section{Introduction}

Image-enhanced endoscopy (IEE), such as narrow-band imaging (NBI), flexible spectral imaging color enhancement (FICE), i-Scan, blue laser imaging (BLI), and autofluorescence imaging (AFI) [1] has been developed in recent years. Accumulating evidence has demonstrated the usefulness of IEE for early detection [2 -4], histological diagnosis including distinguishing between neoplasia and non-neoplasia [5], and estimation of the depth of infiltration [6-8]. 


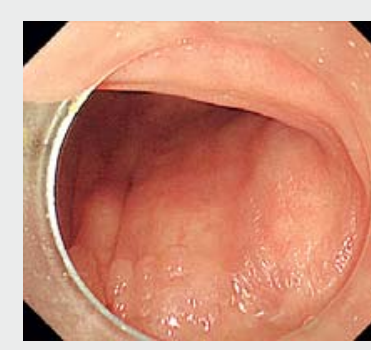

a

WLI

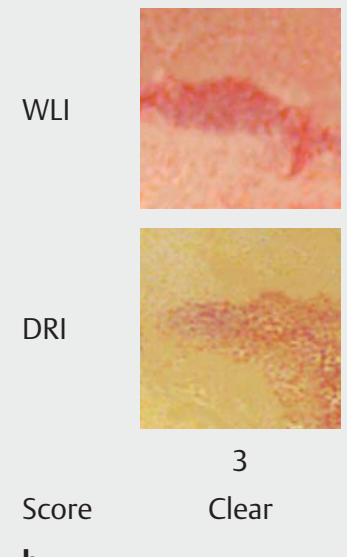

b

- Fig. 1 The appearance of blood vessels and scoring template. a Compared with white light imaging (WLI), dual red imaging (DRI) can clearly detect blood vessels in the deep mucosa or submucosa. b The scores for the visibility of vessels were determined by score.

Most of these IEE technologies use short wavelength light that scatters in the superficial mucosa, thus, most of the targets of these imaging modalities are fine vascular networks and mucosal microstructures within the superficial mucosa. Recently, "dual red imaging" (DRI), a novel IEE technology, has been developed. DRI uses two wavelengths of light in the red band and has the potential to improve the visualization of blood vessels in deeper layers.

This study aimed to confirm the feasibility of DRI in visualization of vessels in deeper tissue and identify pathologically the features of blood vessels visualized by DRI.

\section{Methods}

\section{Study design}

This study comprised a clinical study (study 1) and an animal study using living pigs (study 2 ). In study 1 , we compared the visibility of vessels between conventional white light imaging (WLI) and DRI. In study 2, we analyzed the pathological features of vessels visualized by DRI using a live porcine model. Before initiation of the study, the study protocol was approved by the ethics review board of our animal experimental laboratory (13055-(1)) and the institutional review board of Keio University (No. 20110053). The protocol was also registered with the University Medical Information Network (No.000018351).
Informed consent was obtained from patients included in study 1 .

\section{Principles of DRI}

DRI uses three kinds of illumination light, with central wavelengths of $540 \mathrm{~nm}, 600 \mathrm{~nm}$, and $630 \mathrm{~nm}$. These wavelengths were theoretically optimized to visualize vessels existing in the deep mucosa or submucosa. We used prototype endoscopes based on the EVIS LUCERA SPECTRUM system (CV-Y0012, CLVY0022; Olympus Co., Tokyo, Japan). WLI, DRI, and NBI modes could be switched by pressing a button. The light source unit was equipped with special optical filters for DRI observation without the need for a special scope and contrast agents. Prototype endoscopes (GIF-Y0043, GIF-Y0051, and PCF-Y0029-I) were used. These endoscopes have equivalent specifications to production endoscopes (GIF-Q260), GIF-H260Z, and PCFQ260JI) other than the availability of DRI.

\section{Study 1 (clinical study)}

Five endoscopists, each with more than 5 years of experience in endoscopy, were enrolled. Pairs of DRI and WLI images were randomly taken at the same endoscope position during screening gastroscopy and colonoscopy by senior endoscopists ( $\triangleright$ Fig.1). A small area of a single visible blood vessel was selected from DRI images by a third person. In addition, the same area of paired WLI images was used as a matched image. A total of 137 pairs of DRI and WLI images were selected and used for this study. Those images were randomized and subjected to evaluation without pairing the images. The scores (1-3 scale) for the visibility of blood vessels were determined by reference to the scoring template for all images (score 1: not visible; score 2: visible but not clearly; score 3 : clearly visible) ( $\triangleright$ Fig.1). The difference in visibility score between DRI and WLI was compared in each pair of images.

\section{Study 2 (animal experimental model)}

Two domestic female pigs weighing approximately $25 \mathrm{~kg}$ were used. Both pigs fasted for 24 hours before the interventions. The animals were maintained in the left lateral decubitus position under general anesthesia during all procedures. An endoscope was inserted into the stomach, and mucous was removed by water irrigation. Then the stomach was observed using the DRI mode. After a yellowish vessel had clearly been detected, WLI mode was selected. We carefully determined that a vessel was visible by DRI only when it disappeared under WLI and NBI; two endoclips were deployed next to the vessel. Upon completion of the procedures, the pigs were sacrificed; $5-\mathrm{cm}$ square sections of tissue around the endoclips were resected, pinned onto rubber boards, and fixed in $10 \%$ neutral buffered formalin. Tissues were sliced cross-wise through the centers between the two endoclips, embedded in paraffin, cut into 4- $\mu$ m-thick sections, and treated with hematoxylin and eosin (HE). One pathologist (A. N.) who was blinded to the endoscopic findings examined the histopathologic sections. Vessels with a caliber exceeding $50 \mu \mathrm{m}$ were identified as visible vessels. The depth where these vessels were located and the calibers of identified vessels were noted. 


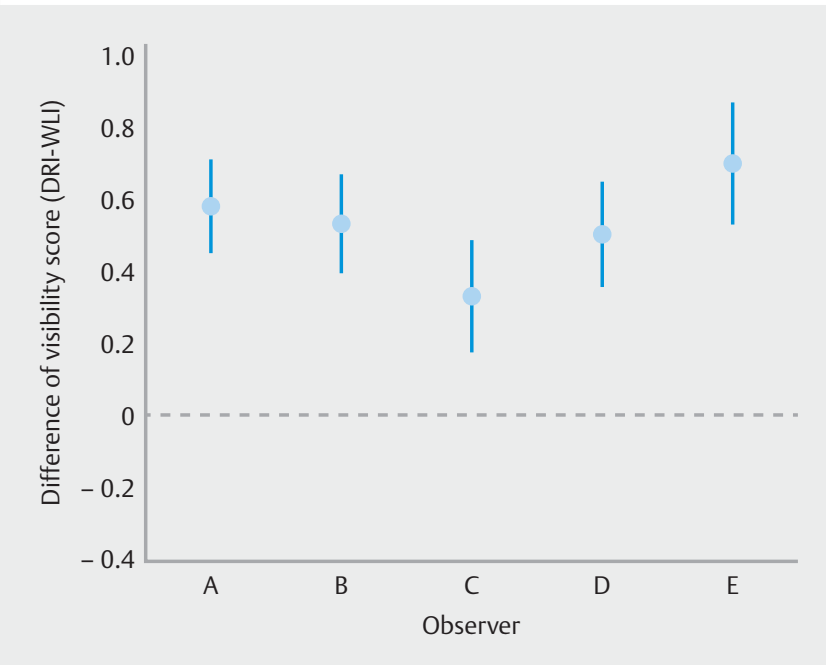

- Fig. 2 The difference in visibility score between DRI and WLI for each observer. Data are shown as mean (circle) and 95\% confidence interval.

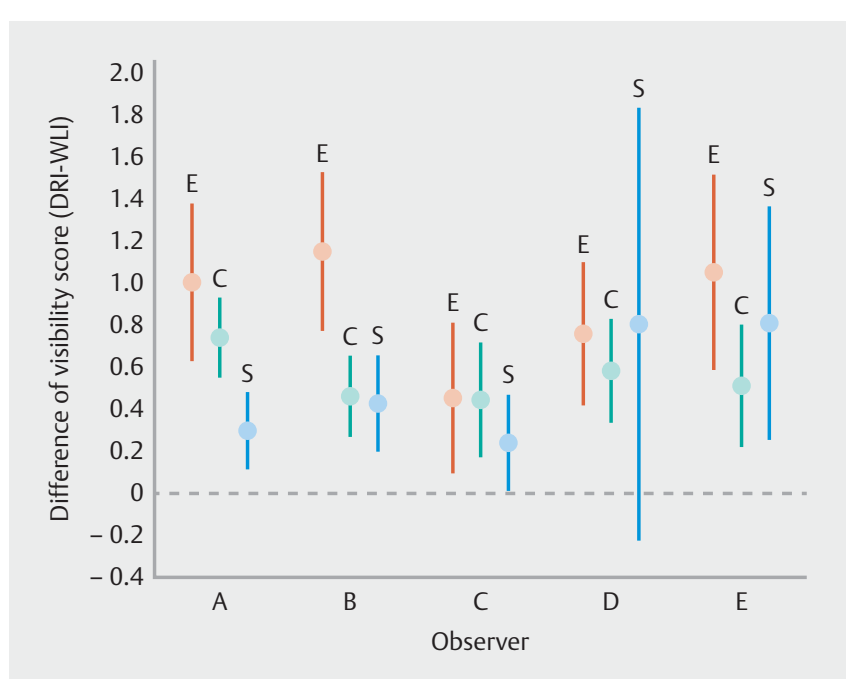

- Fig. 3 The difference in visibility score between DRI and WLI according to organ. Data are shown as mean (circle) and 95\% confidence interval. E: esophagus ( $n=20)$; C: colorectum ( $n=57)$; S: stomach $(n=55)$.

\section{Statistical analysis}

The scores of visibility of vessels were compared between DRI and WLI using the paired $t$ test for each of the five observers. Moreover, a similar analysis was performed in subgroups of the target organ (esophagus, colorectum, and stomach). Data analyses were performed using the JMP program (version 13, SAS Institute, Cary, NC, United States). All $P$ values were two-sided and considered to be significant at values less than 0.05 and Bonferroni's method was used to adjust for multiplicity.

\section{Results}

\section{Study 1 (clinical study)}

The 137 pairs of images consisted of 20 pairs of images of the esophagus, 57 of the colorectum, 55 of the stomach, and 5 of the duodenum. The mean visibility scores of DRI and WLI for each observer were 1.69-2.26 and 1.31-1.67, respectively. The mean differences in visibility score and their $95 \%$ confident intervals for the five observers were 0.59 [0.46-0.72], 0.54 [0.40-0.68], 0.34 [0.18-0.49], 0.51 [0.36-0.66], and 0.71 $[0.54-0.88]$, respectively, and the visibility of vessels was significantly better in DRI than in WLI for all observers $(P<0.0001$, paired $t$ test, $>$ Fig. 2). Next, we analyzed whether this difference might be influenced by different organs. For each observer, we compared the visibility score in subgroups according to the organ. We excluded the five pairs of images of the duodenum, because the number of images was too few to perform statistical analysis. As a result, we found that all subgroups, apart from the stomach subgroup for observer $D$, revealed a significant improvement in visibility of vessels in DRI (॰ Fig. 3 ). The mean differences in visibility score between DRI and WLI in the esophagus, colorectum, and stomach were $0.88 \pm 0.28$, $0.55 \pm 0.12$, and $0.41 \pm 0.22$, respectively. The difference in visibility score in the esophagus was statistically significantly greater than in the stomach $(P=0.018)$, though this was not significant after Bonferroni's adjustment for multiplicity.

\section{Study 2 (animal experiment model)}

Blood vessels were detected in the anterior wall of the middle body of both pigs. Three yellowish blood vessels, including two in pig 1 and one in pig 2, were clearly detected. A histopathologic examination revealed that vessels recognized by DRI had diameters of $800-2000 \mu \mathrm{m}$ and were located approximately $1000-2000 \mu \mathrm{m}$ from the mucosal surface ( $\bullet$ Fig.4). In terms of the kind of vessels, there were both arteries and veins with almost the same caliber of vessels.

\section{Discussion}

In this pilot study, we attempted to assess the feasibility of using DRI for recognition of vessels and to identify the features of blood vessels detected by DRI irrespective of the organ. Our study demonstrated that DRI provided good images of thick blood vessels, which were located 1000-1500 $\mu \mathrm{m}$ from the mucosal surface. The visibility of these thick blood vessels in the deep mucosa or submucosa was significantly better in DRI than in WLI.

With recent advances in endoscopy technologies, a number of IEE techniques have become commercially available [1-8]. However, these technologies focus on fine superficial blood vessels mostly arising from neoplastic lesions and cannot evaluate thick blood vessels in the deep mucosa or submucosa. DRI is a novel IEE technology that has the unique characteristic of utilizing three kinds of illumination light, with central wavelengths of $540 \mathrm{~nm}, 600 \mathrm{~nm}$, and $630 \mathrm{~nm}$. Theoretically, both $600 \mathrm{~nm}$ and $630 \mathrm{~nm}$ light can penetrate deeper tissues because the scattering properties of light with relatively long wavelengths are not strong; moreover, the light absorption by blood 


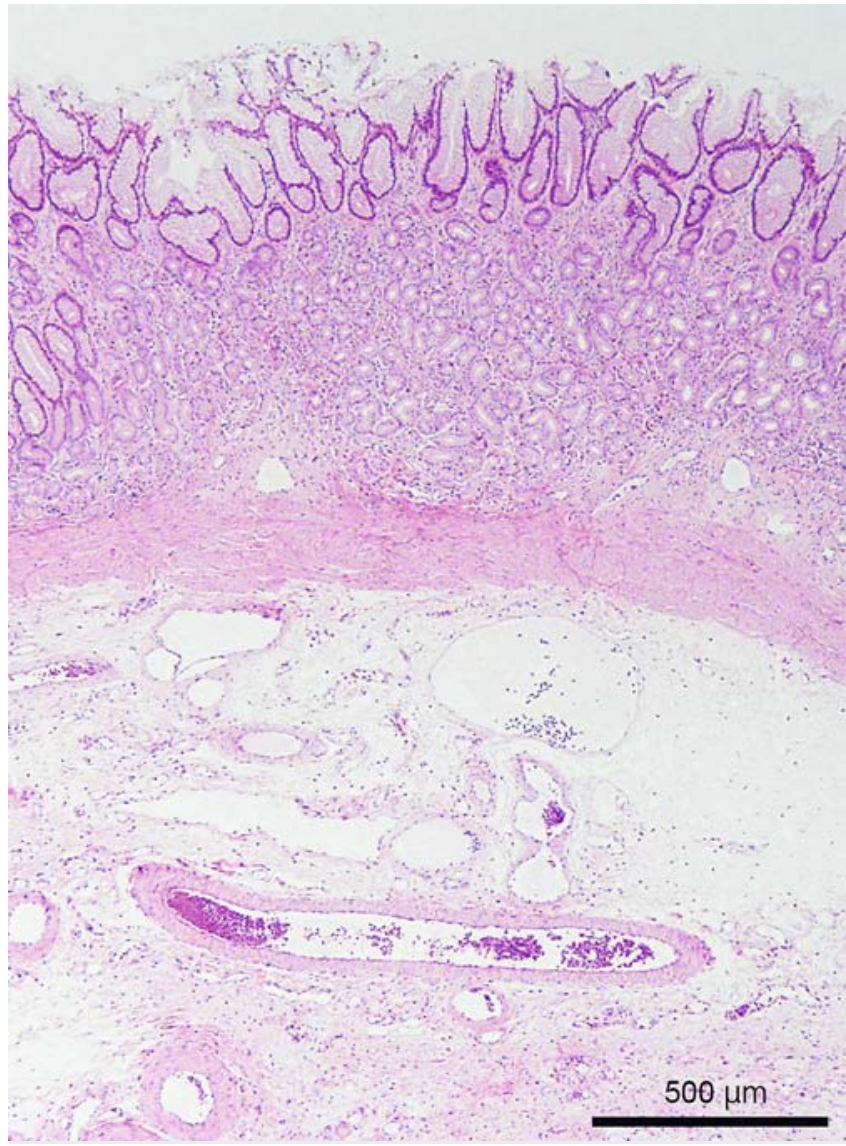

- Fig. 4 Microscopic findings of blood vessels visualized by DRI. Blood vessels with diameters of $800-2000 \mu \mathrm{m}$ were detected at a depth of $1000-2000 \mu \mathrm{m}$ from the mucosal surface.

vessels at $600 \mathrm{~nm}$ is much stronger than that at $630 \mathrm{~nm}$. As a result, the reflected light intensity of $630 \mathrm{~nm}$ light derived from blood vessels is higher than that of $600 \mathrm{~nm}$ light. In contrast to existing IEE technologies, DRI can feasibly visualize thick blood vessels in the deep mucosa and submucosa.

In the subgroup analysis in study 1 , DRI revealed a significant improvement in visibility regardless of organ. This indicates its usefulness and versatility in clinical practice. Moreover, we found the difference in visibility score in the esophagus was greater than in the stomach, though the limited number of images analyzed meant that this difference could not be shown to be statistically significant. In the esophagus, the surface of the lumen is covered with squamous epithelium, which has properties of a thin mucosa and a smooth surface compared to columnar epithelium. Further study is required to elucidate the different visibilities according to organ, and our understanding of anatomical features of the gastrointestinal tract would be enriched through studies using DRI.

Due to the unique characteristics of DRI, this technology could provide further advancements in the understanding of diseases, as well as the development of diagnostic and therapeutic endoscopy by visualizing deep vessels. Recently, we reported the usefulness of DRI in the evaluation of subtle inflammatory changes in ulcerative colitis [9], and other groups have reported the usefulness of DRI in evaluation of esophageal varices [10]. In addition, DRI might be used to visualize neovascular vessels that form after cancer cell infiltration [11-12].

As mentioned above, though our study suggested the potential usefulness of this novel technology, it has some limitations. First, we could not blind observers in study 1, even though the end point was subjective data. Second, the sample size was relatively small in study 2 . Due to these limitations, our results should be interpreted carefully and confirmed by future studies.

In conclusion, we determined that DRI can feasibly detect relatively thick blood vessels at a depth of $1000-2000 \mu \mathrm{m}$ from the surface. DRI could provide further advancements in the understanding of diseases, as well as the development of diagnostic and therapeutic endoscopy by visualizing deep vessels.

\section{Competing interests}

\section{None}

\section{References}

[1] Fujiya M, Kohgo Y. Image-enhanced endoscopy for the diagnosis of colon neoplasms. Gastrointest Endosc 2013; 77: 111-118.e115

[2] Uraoka T, Higashi R, Saito Y et al. Impact of narrow-band imaging in screening colonoscopy. Dig Endosc 2010; 22: S54-S56

[3] Muto M, Minashi K, Yano T et al. Early detection of superficial squamous cell carcinoma in the head and neck region and esophagus by narrow band imaging: a multicenter randomized controlled trial. J Clin Oncol 2010; 28: 1566 - 1572

[4] Kodashima S, Fujishiro M. Novel image-enhanced endoscopy with i-scan technology. World J Gastroenterol 2010; 16: 1043 - 1049

[5] Gupta N, Bansal A, Rao D et al. Accuracy of in vivo optical diagnosis of colon polyp histology by narrow-band imaging in predicting colonoscopy surveillance intervals. Gastrointest Endosc 2012; 75: 494-502

[6] Uraoka T, Saito Y, Ikematsu H et al. Sano's capillary pattern classification for narrow-band imaging of early colorectal lesions. Dig Endosc 2011; 23: $112-115$

[7] Ikematsu H, Matsuda T, Emura F et al. Efficacy of capillary pattern type IIIA/IIIB by magnifying narrow band imaging for estimating depth of invasion of early colorectal neoplasms. BMC Gastroenterol 2010; 10: 33

[8] Sato $\mathrm{H}$, Inoue $\mathrm{H}$, Ikeda $\mathrm{H}$ et al. Utility of intrapapillary capillary loops seen on magnifying narrow-band imaging in estimating invasive depth of esophageal squamous cell carcinoma. Endoscopy 2015; 47: $122-128$

[9] Naganuma M, Yahagi N, Bessho R et al. Evaluation of the severity of ulcerative colitis using endoscopic dual red imaging targeting deep vessels. Endosc Int Open 2017; 5: E76-E82

[10] Furuichi Y, Gotoda T, Moriyasu F et al. Dual red imaging (novel advanced endoscopy) can increase visibility and can predict the depth in diagnosing esophageal varices. J Gastroenterol 2017; 52: 568-576

[11] Fondevila C, Metges JP, Fuster J et al. p53 and VEGF expression are independent predictors of tumour recurrence and survival following curative resection of gastric cancer. Br J Cancer 2004; 90: 206-215

[12] Maeda K, Chung YS, Ogawa Y et al. Prognostic value of vascular endothelial growth factor expression in gastric carcinoma. Cancer 1996; 77: $858-863$ 